\title{
Novel Route for Synthesis of Thiozolidine-2,4-Dione Derivatives as a Mannich Base
}

\author{
Ramakrishna Vellalacheruvu*, Ramayanam Sai Leela, L. K. Ravindranath \\ Department of Chemistry, Sri Krishnadevaraya University, Anantapur, India \\ Email: *vellala143@gmail.com
}

How to cite this paper: Vellalacheruvu, R., Leela, R.S. and Ravindranath, L.K. (2017) Novel Route for Synthesis of Thiozolidine-2,4-Dione Derivatives as a Mannich Base. International Journal of Organic Chemistry, 7, 269-283.

https://doi.org/10.4236/ijoc.2017.73021

Received: July 24, 2017

Accepted: September 3, 2017

Published: September 6, 2017

Copyright $\odot 2017$ by authors and Scientific Research Publishing Inc. This work is licensed under the Creative Commons Attribution International License (CC BY 4.0).

http://creativecommons.org/licenses/by/4.0/

(c) (i) Open Access

\begin{abstract}
The Mannich base of Thiozolidine-2,4-dione derivatives has come to lime light due to their various pharmacological activities. Thiazolidine-2,4-dione is an extensively explored heterocyclic nucleus for designing of novel agents implicated for a wide variety of pathophysiological conditions, that is, diabetes, diabetic complications, cancer, arthritis, inflammation, microbial infection, and melanoma. In present work, synthesis quinoline attached imidazoline derivative using $(3+2)$ cyclo-addition via imine of quinoline and TosMIC. These derivatives were converted to Mannich bases of thiozolidine-2,4-dione using Knoevenagel condensation. The sulfonamide analogues of thiozolidine-2,4-Dione were also synthesized and characterized by using alkylation conditions.
\end{abstract}

\section{Keywords}

Imidazole Analogues, Thiozolidine-2,4-Dione Nucleus,

Toluenesulfonylmethyl Isocyanide, Combi-Flash Column Chromatography

\section{Introduction}

Thiazolidine-2,4-dione (TZD) is a vital nucleus in heterocyclic chemistry. TZD shows multidirectional pharmacological activities such as antioxidant [1], antihyperglycemic (glitazone drugs) [2] [3], antibacterial and anti-fungal [4], anticancer [5], antimicrobial, antitubercular [6], anti-arthritic [7], diabetic \& diabetic complications [8] [9], anti-inflammatory activity [10] [11] and antiplasmodial activity [12]. Due to various pharmacological actions of TZD derivatives, researchers keep on huge interest in synthesis of new TZD derivatives by using various synthetic methods and carry out clinical trials for achieving lead target. Nowadays, diabetes is a major metabolic disorder on human beings throughout 
world. The Thiazolidine-2,4-dione (TZD) derivatives act as drug candidates such as, rosiglitazone, pioglitazone, lobiglitazone, englitazone, metolazone, ozoline, darglitazone and troglitazone etc. TZD derivatives not only confine for treatment of metabolic disorder diabetic, but also show as an inflammatory agent, anti-cancer and for treatment of melanoma. Due to importance of TZD derivatives, many scientists have developed various routes for synthesis.

Om Silakari et al. [2] developed different TZD derivatives and evolutes of their biological activity. The various pharmacological activity of Thiazolidine-2,4-dione was shown in Figure 1.

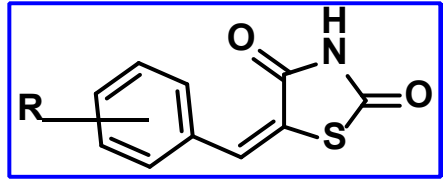

Various approaches have been developed for synthesis of thiazolidine-2,4-dione and there are many active sites for exploring new analogues. Thiazolidine-2,4-dione nucleus numbering is assigned as given below.

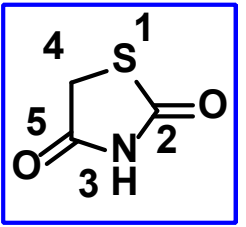

As a part of research work, we synthesize novel Mannich base of thiazolidine-2,4-dione and sulfonamide analogues using an effective and feasible process compared to other approaches and designed to make easy to scale up. We strongly believe these analogues are biologically active.

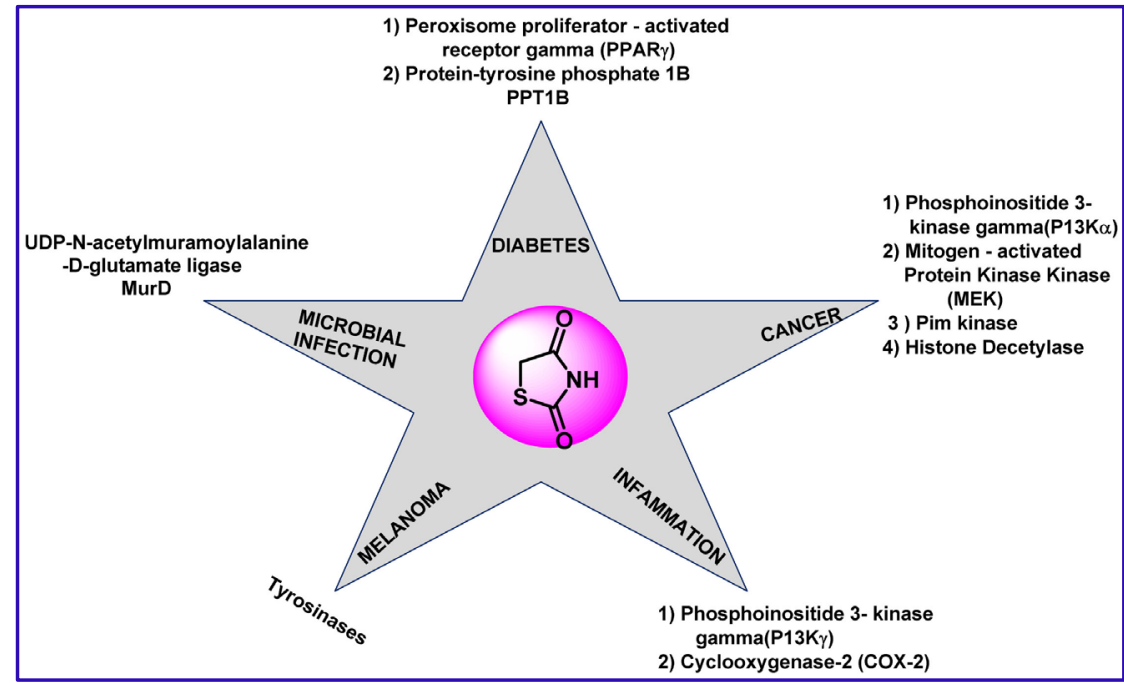

Figure 1. Various pharmacological activity of thiazolidine-2,4-dione. Ivanildo Mangueira da Silva and co workers [3] developed TZD derivatives using Knoevenagel condensation. 


\section{Materials and Methods}

All reagents and starting material were procured from commercial sources (Aldrich, Alfa Aesar) and solvents, such as THF, DMF and toluene, which were thoroughly dried before use. THF and toluene were dried using sodium metal and Benzophenone, and DMF was dried using $\mathrm{CaH}$. The synthesized analogues were fully characterized using Analytical methods like IR, NMR (Bruker). The melting points were recorded using on a WRS-1A digital Melting Point Apparatus without correction. Infrared spectra were taken using an AVATAR 370 FTIR spectrometer. ${ }^{1} \mathrm{HNMR},{ }^{13} \mathrm{CNMR}$ spectra were recorded with a Bruker spectrometer operating at $400 \mathrm{MHz}$ with Trimethylsilane reference and values were recorded in ppm. The progress of reaction was monitored using TLC system and $\mathrm{I}_{2}$ spray and $\mathrm{KMnO}_{4}$ TLC strain. The crude compounds were purified using column chromatography (100 - 200 mesh silica) and Combi Flash Chromatography. The hydrogenolysis process was carried out using parr shaker.

\section{Objective of Research}

Current Research work is related to develop novel synthetic route for synthesis of the Novel mannich bases of thiazolidine-2,4-dione, sulfonamide analogues and thoroughly characterized. The scaffolds of 3-(Amine substituted methyl)5-(2-((5-(1-(4-(trifluoromethyl) phenyl)- H-imidazol-5-yl)quinolin-8-yl) oxy) ethylidene)thiazolidine-2,4-dione (7a-f), and 3-(sulfonyl-derivatives)-5-(2-((5(1-(4-(trifluoromethyl)phenyl)-1H-imidazol-5-yl)quinolin-8-yl)oxy)ethylidene) thiazolidine-2,4-dione (8a-f) were synthesized and characterized.

\section{Experimental Methods}

Current research work, we prepared below compounds and described in step wise manner.

* Step-1: (Z)-N-((8-(benzyloxy)quinolin-5-yl)methylene)-4-(trifluoromethyl) aniline (2).

* Step-2: 8-(benzyloxy)-5-(1-(4-(trifluoromethyl)phenyl)-1H-imidazol-5-yl) quinoline (3).

* Step-3: 5-(1-(4-(trifluoromethyl)phenyl)-1H-imidazol-5-yl)quinolin-8-ol(4).

* Step-4: 2-((5-(1-(4-(trifluoromethyl)phenyl)-1H-imidazol-5-yl)quinolin-8yl)oxy)acetaldehyde (5).

* Step-5: 5-(5-(1-(4-(trifluoromethyl)phenyl)-1H-imidazol-5-yl)quinolin-8-yl) oxy)methylene)thiazolidine-2,4-dione (6).

* Step-6: 3-(Amine substituted methyl)-5-(2-((5-(1-(4-(trifluoromethyl) phenyl)-1H-imidazol-5-yl)quinolin-8-yl)oxy)ethylidene)thiazolidine-2,4-dio ne (7a-f).

* Step-7: 3-(sulfonyl-derivatives)-5-(2-((5-(1-(4-(trifluoromethyl)phenyl)-1Himidazol-5-yl)quinolin-8-yl)oxy)ethylidene)thiazolidine-2,4-dione (8a-f). The Reaction scheme was shown in Scheme 1. The Reaction mechanism of Step 2 was shown in Figure 2. 


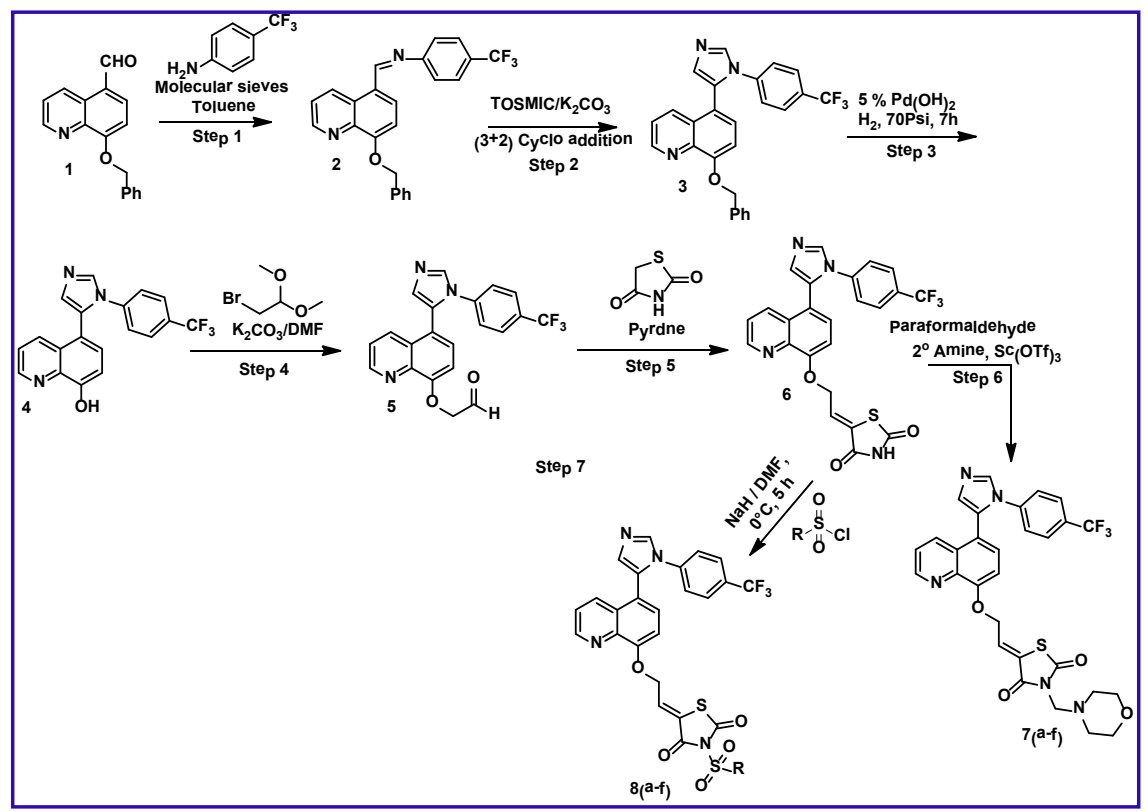

Scheme 1. Synthesis of 3-(Amine substituted methyl)-5-(2-((5-(1-(4-(trifluoromethyl) phenyl)-1H-imidazol-5-yl)quinolin-8-yl)oxy)ethylidene)thiazolidine-2,4-dione(7a-f) \& sulphonamide analogues $8(\mathrm{a}-\mathrm{f})$.

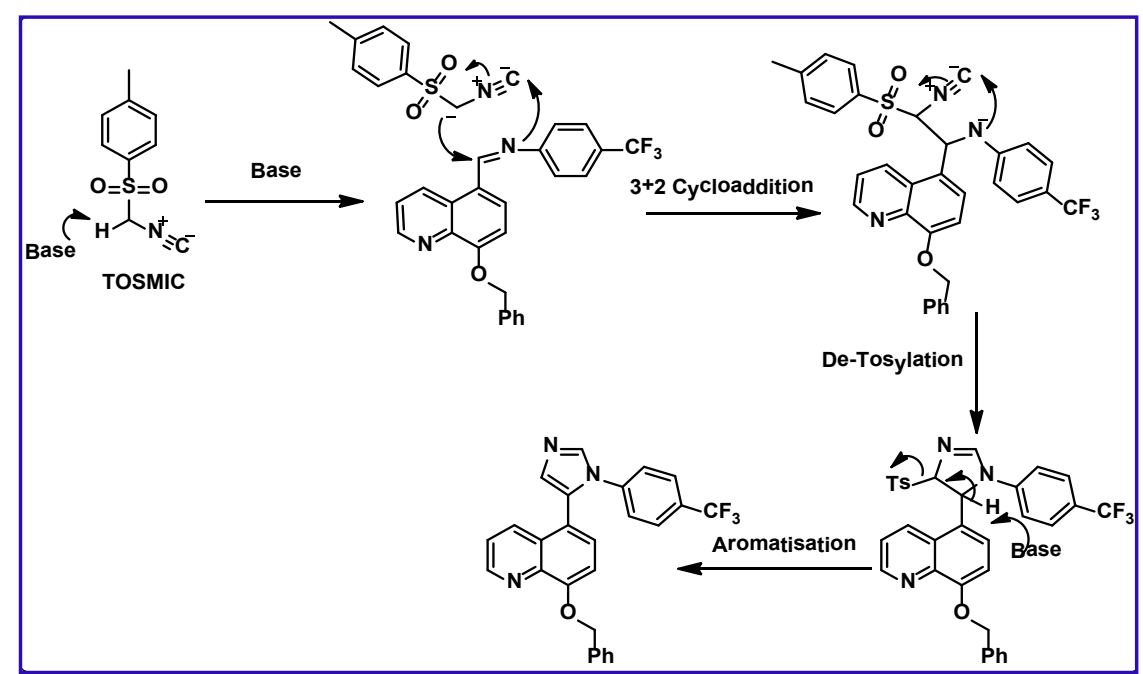

Figure 2. The Reaction mechanism for step 2.

\section{Scheme}

Reaction Conditions: Step 1: Molecular sieves, Toluene, $0^{\circ} \mathrm{C}$, $10 \mathrm{~h}$, Step 2: TOSMIC $/ \mathrm{K}_{2} \mathrm{CO}_{3}, 0^{\circ} \mathrm{C}, 16 \mathrm{~h}$, Step 3: $5 \% \mathrm{Pd}(\mathrm{OH})_{2}, \mathrm{H}_{2}, 70$ Psi, $3 \mathrm{~h}$, Step 4: 2 bromo-1,1-dimethoxyethane, $\mathrm{K}_{2} \mathrm{CO}_{3} / \mathrm{DMF}, 5 \mathrm{~h}$, Step 5: thiazolidine-2,4-dione, Piperdine, $90^{\circ} \mathrm{C}, 6 \mathrm{~h}$, Step 6: Paraformaldehyde $/ 2^{\circ}$ Amine, $\mathrm{Sc}(\mathrm{OTf})_{3}, \mathrm{EtOH}$, $90^{\circ} \mathrm{C}, 8 \mathrm{~h}$. Step 7: $\mathrm{NaH}, \mathrm{DMF}, 0^{\circ} \mathrm{C}, 5 \mathrm{~h}$.

Reaction mechanism for Step 2: The Reaction mechanism of step 2 was shown in Figure 2.

Step 1: (Z)-N-((8-(benzyloxy)quinolin-5-yl)methylene)-4-(trifluoromethyl)aniline(2): 


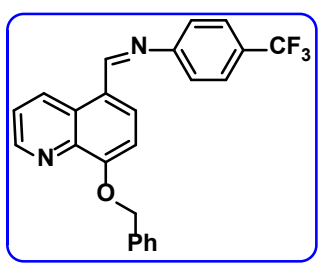

8-(benzyloxy)quinoline-5-carbaldehyde ( $10 \mathrm{~g}, 0.038 \mathrm{~mol}), 4$-(trifluoromethyl) aniline $(6.5 \mathrm{~g}, 0.039 \mathrm{~mol})$ in dry toluene $(100 \mathrm{~mL})$ was added freshly dried molecular sieves and refluxed for $10 \mathrm{~h}$ under $\mathrm{N}_{2}$ atm. The progress of reaction was monitored by TLC. After completion of starting material, toluene was evaporated under vacuum to give crude residue of Compound-2 $(15 \mathrm{~g})$ as a solid. The crude was carried to next step.

Step 2: 8-(benzyloxy)-5-(1-(4-(trifluoromethyl)phenyl)-1H-imidazol-5yl)quinoline(3):

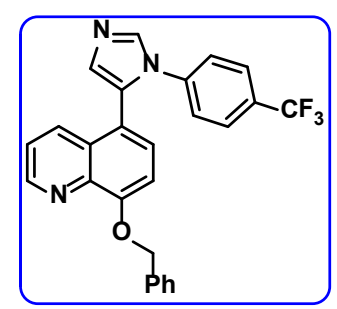

(Z)-N-((8-(benzyloxy)quinolin-5-yl)methylene)-4-(trifluoromethyl)aniline(2) $(15 \mathrm{~g}, 0.036 \mathrm{~mol})$ was dissolved in Dry DMF $(80 \mathrm{~mL})$ and cooled to $0{ }^{\circ} \mathrm{C}$. To that dried $\mathrm{K}_{2} \mathrm{CO}_{3}(15 \mathrm{~g} .108 \mathrm{~mol})$ and Toluene methyl isocyanide $(7.02 \mathrm{~g}, 0.036 \mathrm{~mol})$ was added and warm to room temperature and stirred for $16 \mathrm{~h}$. The progress of reaction was monitored by TLC. After completion, reaction mixture was poured in ice cold water $(100 \mathrm{~mL})$ and extracted with EtOAc $(3 \times 100 \mathrm{~mL})$. The organic layer was separated and washed with brine solution, dried over anhydrous $\mathrm{Na}_{2} \mathrm{SO}_{4}$, filtered and evaporated under vacuum to give crude residue. The obtained crude product was purified by column chromatography (100 - 200 mesh silica, Eluent: 80\% EtOAc-Pet Ether) isolated 8-(benzyloxy)-5-(1-(4-(trifluoromethyl)phenyl)-1H-imidazol-5-yl)quinoline (3) (10 g, yield: $64 \%)$ as a solid (white). M.p: $252^{\circ} \mathrm{C}-255^{\circ} \mathrm{C}$. IR $\left(\mathrm{KBr}, \mathrm{cm}^{-1}\right): 3030,1440,1520,1005,691,655$. ${ }^{1} \mathrm{HNMR}\left(d_{6}\right.$-DMSO, $\left.400 \mathrm{MHz}\right): 5.2(\mathrm{~s}, 2 \mathrm{H}), 7.1(\mathrm{~m}, 2 \mathrm{H}), 7.3$ - $7.5(\mathrm{~m}, 7 \mathrm{H}), 7.6$ $7.7(\mathrm{~m}, 4 \mathrm{H}), 7.85(\mathrm{~d}, 1 \mathrm{H}), 8.36(\mathrm{~d}, 1 \mathrm{H}), 8.85(\mathrm{~d}, 1 \mathrm{H})$.

Step 3: 5-(1-(4-(trifluoromethyl)phenyl)-1H-imidazol-5-yl)quinolin-8-ol (4):

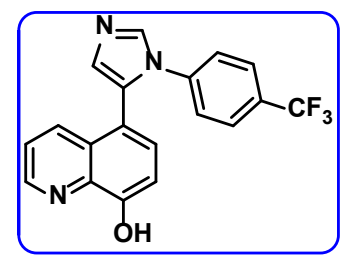


8-(benzyloxy)-5-(1-(4-(trifluoromethyl)phenyl)-1H-imidazol-5-yl)quinoline( 3) $(10 \mathrm{~g}, 0.022 \mathrm{~mol})$ in $\mathrm{MeOH}(100 \mathrm{~mL})$ was added $5 \%$ Palladium hydroxide on carbon ( $1 \mathrm{~g}$, cat) and carried out hydrogenolysis at 70 Psi under parr shaker for 3 $\mathrm{h}$ at room temperature. The progress of reaction was monitored by TLC. After completion, reaction mixture was filtered on celite bed and thoroughly washed with $\mathrm{MeOH}(2 \times 75 \mathrm{~mL})$. The $\mathrm{MeOH}$ layer were collected and evaporated under vacuum to give 5-(1-(4-(trifluoromethyl)phenyl)-1H-imidazol-5-yl)quinolin-8ol (4) (7 g, yield: $86 \%$ ) as a solid(pale white). M.p: $280^{\circ} \mathrm{C}-285^{\circ} \mathrm{C}$. IR $\left(\mathrm{KBr}, \mathrm{cm}^{-1}\right)$ : 3620, 3014, 1525, 1050, 691, 620. ${ }^{1} \mathrm{HNMR}$ (d -DMSO, $400 \mathrm{MHz}$ ): 6.5 (brs, 1H), $7.1(\mathrm{~m}, 2 \mathrm{H}), 7.3(\mathrm{~d}, 2 \mathrm{H}), 7.63(\mathrm{~m}, 4 \mathrm{H}), 7.8(\mathrm{~d}, 1 \mathrm{H}), 8.35(\mathrm{~d}, 1 \mathrm{H}), 8.8(\mathrm{~d}, 1 \mathrm{H})$.

Step 4: 2-((5-(1-(4-(trifluoromethyl)phenyl)-1H- imidazol-5-yl)quinolin8-yl)oxy) acetaldehyde (5):

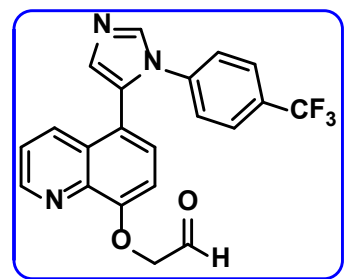

5-(1-(4-(trifluoromethyl)phenyl)-1H-imidazol-5-yl)quinolin-8-ol (4) (7 g, $0.017 \mathrm{~mol})$ in Dry DMF $(70 \mathrm{~mL})$ was added $\mathrm{K}_{2} \mathrm{CO}_{3}(9.7 \mathrm{~g}, 0.07 \mathrm{~mol}, 4 \mathrm{eq})$ and stirred at $\mathrm{rt}$ for $30 \mathrm{~min}$. To that a solution of 2-bromo-1, 1-dimethoxyethane (1.2 eq) in DMF $(20 \mathrm{~mL})$ was added dropwise at $0^{\circ} \mathrm{C}$ and stirred for $5 \mathrm{~h}$. The progress of reaction was monitored by TLC. After completion, reaction mixture was filtered on celite bed and washed with DMF $(10 \mathrm{~mL})$. The Reaction mixture was poured in ice cold water $(200 \mathrm{~mL})$ and stirred for $20 \mathrm{~min}$. The reaction mixture was acidified with aqNaHSO${ }_{3}$ solution up to $\mathrm{P}^{\mathrm{H}}-5$ and extracted with EtOAc (2 $\times 200 \mathrm{~mL}$ ). The aqueous layer was collected and basified up to $\mathrm{P}^{\mathrm{H}}-8$ with sat aq$\mathrm{NaHCO}_{3}$ sol. The aqueous layer was extracted with EtOAc $(3 \times 100 \mathrm{~mL})$. The organic layer were collected and dried over anhydrous $\mathrm{Na}_{2} \mathrm{SO}_{4}$, filtered and evaporated under vacuum to give 2-((5-(1-(4-(trifluoromethyl)phenyl)-1H-imidazol-5-yl)quinolin-8-yl)oxy) acetaldehyde (5) (5 g). M.p: $200^{\circ} \mathrm{C}-205^{\circ} \mathrm{C}$, IR (KBr, $\mathrm{cm}^{-1}$ ): 3602, 3014, 1712, 1646, 1503, 1050, 691, 644. ${ }^{1} \mathrm{HNMR}$ ( $d_{6}$-DMSO, 400 MHz): 5.2 (s, 2H), 7.1 (m, 2H), $7.3(\mathrm{~d}, 2 \mathrm{H}), 7.6-7.65(\mathrm{~m}, 4 \mathrm{H}), 7.9(\mathrm{~d}, 1 \mathrm{H}), 8.36$ $(\mathrm{d}, 1 \mathrm{H}), 8.82(\mathrm{~d}, 1 \mathrm{H}), 9.6(\mathrm{~s}, 1 \mathrm{H})$.

Step 5: 5-(2-((5-(1-(4-(trifluoromethyl)phenyl)-1H-imidazol-5-yl)quinolin-8-yl)oxy) ethylidene) thiazolidine-2,4-dione (5):

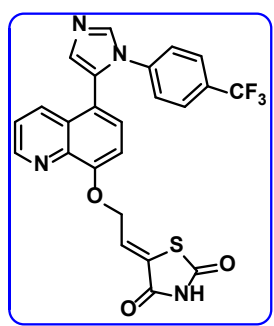


To a mixture of 2-((5-(1-(4-(trifluoromethyl)phenyl)-1H-imidazol-5-yl)quinolin-8-yl)oxy) acetaldehyde (5) (5 g, $0.012 \mathrm{~mol})$, thiazolidine-2,4-dione (1.62 g, $0.013 \mathrm{~mol})$ in EtOH $(50 \mathrm{~mL})$ was added Piperdine $(2 \mathrm{~mL})$ and heated at $90^{\circ} \mathrm{C}$ for $6 \mathrm{~h}$. The progress of reaction was monitored by TLC. After completion, Reaction mixture was evaporated under vacuum to give crude residue. The residue was dissolved in water $(100 \mathrm{~mL})$ and filtered under vacuum and dried to give $(\mathrm{Z})$-5(2-((5-(1-(4-(trifluoromethyl) phenyl)-1H-imidazol-5-yl)quinolin-8-yl)oxy) ethy-lidene) thiazolidine-2,4-dione (6) (5.5 g, Yield: $88 \%$ ) as a solid( red color). M.p:240-243 ${ }^{\circ} \mathrm{C}$. IR $\left(\mathrm{KBr}, \mathrm{cm}^{-1}\right): 3050,1725,1650,1503,1050,691,644 .{ }^{1} \mathrm{HNMR}$ (d d $\left.^{-\mathrm{DMSO}}, 400 \mathrm{MHz}\right): 4.6(\mathrm{dd}, 1 \mathrm{H}), 4.61(\mathrm{dd}, 1 \mathrm{H}), 6.15(\mathrm{dd}, 1 \mathrm{H}), 7.12(\mathrm{~m}, 2 \mathrm{H})$, $7.3(\mathrm{~d}, 2 \mathrm{H}), 7.6$ - $7.65(\mathrm{~m}, 4 \mathrm{H}), 7.9(\mathrm{~d}, 1 \mathrm{H}), 8.4(\mathrm{~d}, 1 \mathrm{H}), 8.6$ (brs, $1 \mathrm{H}), 8.87$ (d, $1 \mathrm{H})$.

Step 6: 3-(Amino substituted methyl)-5-(2-((5-(1-(4-(trifluoromethyl) phenyl)-1H-imidazol-5-yl)quinolin-8-yl)oxy)ethylidene)thiazolidine-2,4-di one \& $7(\mathrm{a}-\mathrm{f})$ :

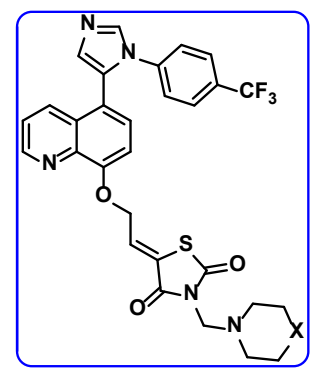

To a mixture of (Z)-5-(2-((5-(1-(4-(trifluoromethyl) phenyl)-1H-imidazol5-yl)quino-lin-8-yl)oxy)ethylidene) thiazolidine-2,4-dione (6) (500 mg), $2^{\circ}$ Amine (1.1 eq), Para formaldehyde ( 3 eq) in $\mathrm{EtOH}(50 \mathrm{~mL})$ was added $\mathrm{Sc}(\mathrm{OTf})_{3}(0.1 \mathrm{eq})$ and heated for $8-12 \mathrm{~h}$. The progress of reaction was monitored by TLC. After completion, EtOH was evaporated under vacuum to give crude product. The crude was purified by reverse-phase column chromatography (C18 silica, eluent: $30 \%$ ACN-MeOH- $\mathrm{H}_{2} \mathrm{O}, 0.01 \%$ TFA) isolated (Z)-3-(Amino-substituted, methyl)-5-(2-((5-(1-(4-(trifluoromethyl)phenyl)-1H-imidazol-5-yl)quinolin-8-yl)o xy)ethylidene) thiazolidine-2,4-dione 7 (a-f).The summary of physical data for analogues 7 (a-f) was shown in Table 1.

3-((4-oxopiperidin-1-yl)methyl)-5-(2-((5-(1-(4-(trifluoromethyl)phenyl)1H-imidazol-5-yl)quinolin-8-yl)oxy)ethylidene)thiazolidine-2,4-dione (7a):

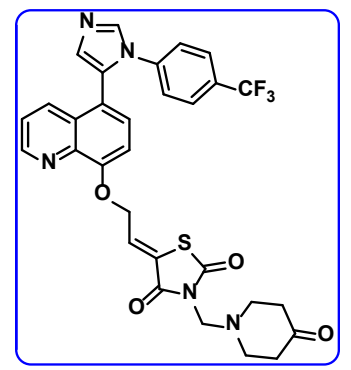


Table 1. Summary of physical data for analogues $7(a-f)$.

\begin{tabular}{ccccccc}
\hline Comp & $7 \mathrm{a}$ & $7 \mathrm{~b}$ & $7 \mathrm{c}$ & $7 \mathrm{~d}$ & $7 \mathrm{e}$ & $7 \mathrm{f}$ \\
\hline $\mathrm{X}$ & $\mathrm{C}=\mathrm{O}$ & $\mathrm{N}-\mathrm{Me}$ & $\mathrm{N}-\mathrm{Boc}$ & $\mathrm{N}-\mathrm{Et}$ & $\mathrm{O}$ & $\mathrm{S}$ \\
Yield & $50 \%$ & $75 \%$ & $70 \%$ & $78 \%$ & $68 \%$ & $70 \%$ \\
Reaction Time & $12 \mathrm{~h}$ & $8 \mathrm{~h}$ & $9 \mathrm{~h}$ & $8 \mathrm{~h}$ & $10 \mathrm{~h}$ & $10 \mathrm{~h}$ \\
\hline
\end{tabular}

M.p: $280^{\circ} \mathrm{C}-283^{\circ} \mathrm{C}$. IR (KBr, $\left.\mathrm{cm}^{-1}\right): 3050,3010,1720,1655,1600,1320,770$, 620. ${ }^{1} \mathrm{HNMR}\left(\boldsymbol{d}_{6}\right.$-DMSO, $\left.400 \mathrm{MHz}\right): 2.4(\mathrm{t}, 4 \mathrm{H}), 2.8(\mathrm{t}, 4 \mathrm{H}), 4.68(\mathrm{dd}, 2 \mathrm{H}), 4.7$ $(\mathrm{dd}, 1 \mathrm{H}), 4.9(\mathrm{~s}, 2 \mathrm{H}), 6.8(\mathrm{dd}, 1 \mathrm{H}), 7.1(\mathrm{~m}, 2 \mathrm{H}), 7.3(\mathrm{~d}, 2 \mathrm{H}), 7.6(\mathrm{~m}, 4 \mathrm{H}), 8.0(\mathrm{~d}$, $1 \mathrm{H}), 8.43(\mathrm{~d}, 1 \mathrm{H}), 8.8(\mathrm{~d}, 1 \mathrm{H}) .{ }^{13} \mathrm{CNMR}$ ( $d_{6}$-DMSO, $\left.400 \mathrm{MHz}\right): 45,53,65,108$, $120,122.9,123.5,124,124.5,126.7,131,135,139,139.2,145,150,155,164,173$, and 190 .

3-((4-methylpiperazin-1-yl)methyl)-5-(2-((5-(1-(4-(trifluoromethyl)phen yl)-1H-imidazol-5-yl)quinolin-8-yl)oxy)ethylidene)thiazolidine-2,4-dione (7b):

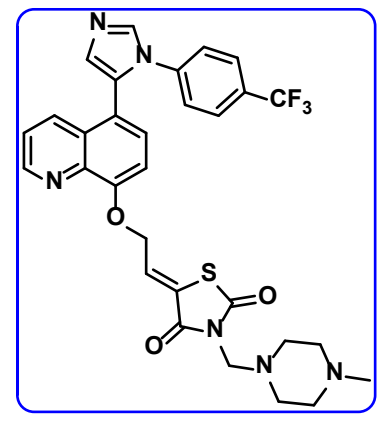

M.p. $290^{\circ} \mathrm{C}-292^{\circ} \mathrm{C} . \mathrm{IR}\left(\mathrm{KBr}, \mathrm{cm}^{-1}\right): 3350,3050,1660,1610,1320,750,625$, ${ }^{1}$ HNMR ( $d_{6}$-DMSO, $400 \mathrm{MHz}$ ): 2.3 (s, 3H), $2.4(\mathrm{~d}, 4 \mathrm{H}), 2.45$ (d, 4H), 4.63 (s, $2 \mathrm{H},), 4.66(\mathrm{dd}, 1 \mathrm{H}), 4.67(\mathrm{dd}, 1 \mathrm{H}), 6.81(\mathrm{dd}, 1 \mathrm{H}), 7.1(\mathrm{~m}, 2 \mathrm{H}), 7.32(\mathrm{~d}, 2 \mathrm{H}), 7.6$ $(\mathrm{m}, 4 \mathrm{H}), 8.03(\mathrm{~d}, 1 \mathrm{H}), 8.44(\mathrm{~d}, 1 \mathrm{H}), 8.81(\mathrm{~d}, 1 \mathrm{H}) .{ }^{13} \mathrm{C}-\mathrm{NMR}\left(\boldsymbol{d}_{6}-\mathrm{DMSO}, 400\right.$ MHz): 47, 53, 58, 65, 107, 121, 122, 123.8, 124, 124.5, 125.5, 127, 130, 134, 138, $138.8,145,150,155.5,163,174$.

tert-butyl 4-((2,4-dioxo-5-(2-((5-(1-(4-(trifluoromethyl)phenyl)-1H- imidazol-5-yl)qu -in olin-8-yl)oxy)ethylidene)thiazolidin-3-yl) methyl)piperazine-1-carboxylate (7c):

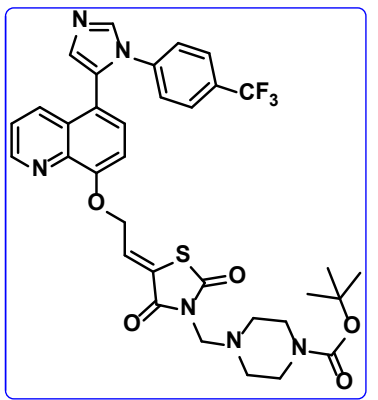


M.p: $260^{\circ} \mathrm{C}-2262^{\circ} \mathrm{C}$. IR $\left(\mathrm{KBr}, \mathrm{cm}^{-1}\right): 3014,1713,1650,1620,1505,1310$, 1050, 698, 655. ${ }^{1} \mathrm{HNMR}$ ( $\boldsymbol{d}_{6}$-DMSO, $\left.400 \mathrm{MHz}\right): 1.4(\mathrm{~s}, 9 \mathrm{H}), 2.5(\mathrm{t}, 4 \mathrm{H}), 3.1(\mathrm{t}$, $4 \mathrm{H}), 2.45(\mathrm{~d}, 4 \mathrm{H}), 4.5(\mathrm{~s}, 2 \mathrm{H}), 4.68(\mathrm{dd}, 1 \mathrm{H}), 4.69(\mathrm{dd}, 1 \mathrm{H}), 6.83(\mathrm{dd}, 1 \mathrm{H}), 7.1(\mathrm{~m}$, 2H), 7.32 (d, 2H), $7.62(\mathrm{~m}, 4 \mathrm{H}), 7.9(\mathrm{~d}, 1 \mathrm{H}), 8.42(\mathrm{~d}, 1 \mathrm{H}), 8.82(\mathrm{~d}, 1 \mathrm{H}) .{ }^{13} \mathrm{C}-\mathrm{NMR}$ (d6-DMSO, $400 \mathrm{MHz}): 31,44,52,65,78,107,121,124,124.5,125.4,127,130$, $132,135.5,138.5,139,145,149,154,162,174$.

3-((4-ethylpiperazin-1-yl)methyl)-5-(2-((5-(1-(4-(trifluoromethyl)phen yl)-1H-imidazol-5-yl)quinolin-8-yl)oxy)ethylidene)thiazolidine-2,4-dione (7d):

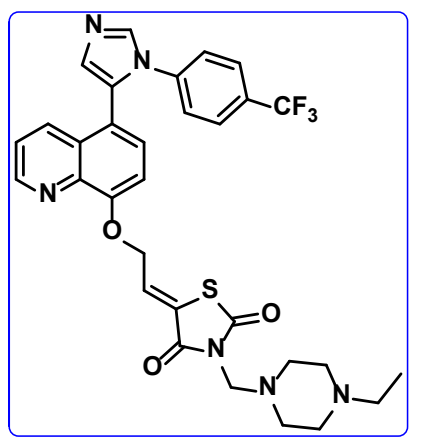

M.p: $290^{\circ} \mathrm{C}-292^{\circ} \mathrm{C}$. IR (KBr, cm $\left.{ }^{-1}\right): 3350,3020,1680,1620,1330,750,625$, ${ }^{1} \mathrm{HNMR}\left(d_{6}\right.$-DMSO, $\left.400 \mathrm{MHz}\right): 1.2(\mathrm{t}, 3 \mathrm{H}), 2.5(\mathrm{~m}, 10 \mathrm{H}), 4.5(\mathrm{~s}, 2 \mathrm{H}), 4.67(\mathrm{~d}$, $1 \mathrm{H}), 4.68(\mathrm{~d}, 1 \mathrm{H}), 6.80(\mathrm{dd}, 1 \mathrm{H}), 7.1(\mathrm{~m}, 2 \mathrm{H}), 7.3(\mathrm{~d}, 2 \mathrm{H}), 7.62(\mathrm{~m}, 4 \mathrm{H}), 7.8(\mathrm{~d}$, $1 \mathrm{H}), 8.42(\mathrm{~d}, 1 \mathrm{H}), 8.82(\mathrm{~d}, 1 \mathrm{H}) .{ }^{13} \mathrm{C}-\mathrm{NMR}$ ( $d_{6}$-DMSO, $\left.400 \mathrm{MHz}\right): 14,50,53,58$, 65, 108, 122, 123.9, 124, 124.1, 125, 130, 132, 135, 139, 145, 149, 155, 165, 174.

3-(morpholinomethyl)-5-(2-((5-(1-(4-(trifluoromethyl)phenyl)-1H-imid azol-5-yl)quinolin-8-yl)oxy)ethylidene)thiazolidine-2,4-dione (7e):

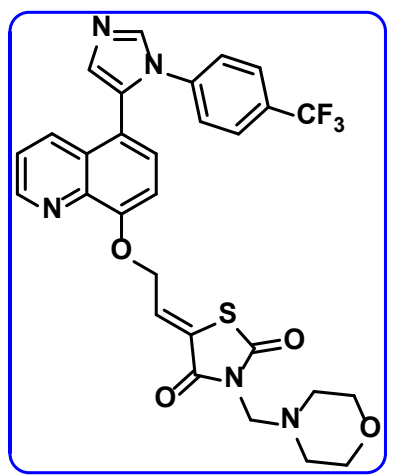

M.p: $280^{\circ} \mathrm{C}-282^{\circ} \mathrm{C}$. IR $\left(\mathrm{KBr}, \mathrm{cm}^{-1}\right): 3016,1720,1650,1503,1300,1050,695$, 650. ${ }^{1} \mathrm{HNMR}\left(\boldsymbol{d}_{6}\right.$-DMSO, $\left.400 \mathrm{MHz}\right): 2.6(\mathrm{t}, 4 \mathrm{H}), 3.7(\mathrm{t}, 4 \mathrm{H}), 4.5(\mathrm{~s}, 2 \mathrm{H}), 4.68$ $(\mathrm{dd}, 1 \mathrm{H}), 4.69(\mathrm{dd}, 1 \mathrm{H}), 6.80(\mathrm{dd}, 1 \mathrm{H}), 7.15(\mathrm{~m}, 2 \mathrm{H}), 7.25(\mathrm{~d}, 2 \mathrm{H}), 7.62(\mathrm{~m}$, $4 \mathrm{H}), 7.9(\mathrm{~d}, 1 \mathrm{H}), 8.38(\mathrm{~d}, 1 \mathrm{H}), 8.89(\mathrm{~d}, 1 \mathrm{H}) .{ }^{13} \mathrm{C}-\mathrm{NMR}\left(d_{6}\right.$-DMSO, $\left.400 \mathrm{MHz}\right)$ : $53,65,66,107,121.9,123.8,124,124.5,127,130,132,135.5,139,145,149,155$, 164, 176. 
3-(thiomorpholinomethyl)-5-(2-((5-(1-(4-(trifluoromethyl)phenyl)-1H-i midazol-5-yl)quinolin-8-yl)oxy)ethylidene)thiazolidine-2,4-dione (7f):

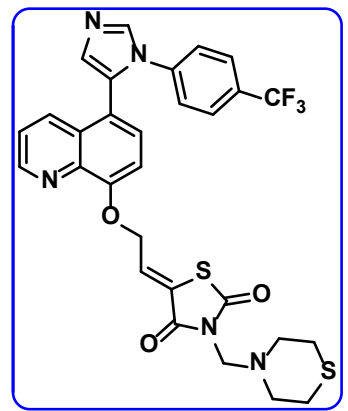

M.p: $272^{\circ} \mathrm{C}-275^{\circ} \mathrm{C} . \mathrm{IR}\left(\mathrm{KBr}, \mathrm{cm}^{-1}\right): 3080,1730,1645,1520,1310,1125,670$, 660, ${ }^{1} \mathrm{HNMR}\left(d_{6}\right.$-DMSO, $\left.400 \mathrm{MHz}\right): 2.6(\mathrm{t}, 4 \mathrm{H}), 2.8(\mathrm{t}, 4 \mathrm{H}), 4.51(\mathrm{~s}, 2 \mathrm{H}), 4.66$ (dd, 1H), $4.67(\mathrm{dd}, 1 \mathrm{H}), 6.81(\mathrm{dd}, 1 \mathrm{H}), 7.15(\mathrm{~m}, 2 \mathrm{H}), 7.25(\mathrm{~d}, 2 \mathrm{H}), 7.62(\mathrm{~m}, 4 \mathrm{H})$, $7.9(\mathrm{~d}, 1 \mathrm{H}), 8.37(\mathrm{~d}, 1 \mathrm{H}), 8.86(\mathrm{~d}, 1 \mathrm{H}) .{ }^{13} \mathrm{C}-\mathrm{NMR}\left(d_{6}\right.$-DMSO, $\left.400 \mathrm{MHz}\right): 27,58$, $63,106,122,123,124,124.5,125,130,131,135,138.5,139,145,149,155.4,164$, 174 .

Step 7: General procedure for -3-(sulfonyl derivative)-5-(2-((5-(1-(4(trifluoromethyl)phenyl)-1H-imidazol-5-yl)quinolin-8-yl)oxy)ethylidene) thiazolidine-2,4-dione (8a-f):

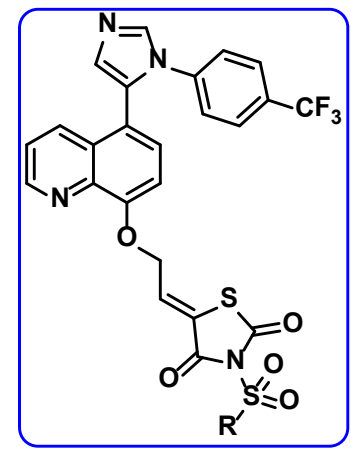

5-(2-((5-(1-(4-(trifluoromethyl)phenyl)-1H-imidazol-5-yl)quinolin-8-yl)oxy) ethylidene) thiazolidine-2,4-dione (500 $\mathrm{mg}, 1.08 \mathrm{mmol}$ ) in Dry DMF $(5 \mathrm{~mL})$ was added $\mathrm{NaH}$ ( 3 eq) at $0^{\circ} \mathrm{C}$ under $\mathrm{N}_{2}$ atm and stirred for $1 \mathrm{~h}$. To that derivative of sulfonyl chloride (1.1 eq) was added and stirred for $5-8 \mathrm{~h}$. The progress of reaction was monitored by TLC. The reaction mixture was poured in aq sat $\mathrm{NaHCO}_{3}$ and stirred for $15 \mathrm{~min}$. The a.q layer was extracted with $10 \% \mathrm{MeOH}_{-} \mathrm{CHCl}_{3}(3 \times$ $25 \mathrm{ml}$ ) and dried over anhydrous $\mathrm{Na}_{2} \mathrm{SO}_{4}$, filtered and evaporated under vacuum to give crude product. The crude product was purified by Column chromatography (100 - 200 mesh silica) isolated 3-(sulfonyl derivative)-5-(2-((5-(1-(4(trifluoromethyl) phenyl)-1H-imidazol-5-yl)quinolin-8-yl)oxy) ethylidene) thiazolidine-2,4-dione (8a-f). The summary of physical data for analogues is shown in Table 2. 
Table 2. Summary table for analogues $8(a-f)$.

\begin{tabular}{ccccccc}
\hline \multicolumn{7}{c}{ Analogues } \\
\hline Comp & $8 \mathrm{a}$ & $8 \mathrm{~b}$ & $8 \mathrm{c}$ & $8 \mathrm{~d}$ & $8 \mathrm{e}$ & $8 \mathrm{f}$ \\
\hline $\mathrm{R}$ & $\mathrm{Me}$ & $\mathrm{Et}$ & $\mathrm{P}-\mathrm{Tolyl}$ & $4-\mathrm{Cl}_{-} \mathrm{C}_{6} \mathrm{H}_{4}$ & $4-\mathrm{Br}_{-} \mathrm{C}_{6} \mathrm{H}_{4}$ & $4-\mathrm{NO}_{2}-\mathrm{C}_{6} \mathrm{H}_{4}$ \\
Reaction Time & $5 \mathrm{~h}$ & $5 \mathrm{~h}$ & $8 \mathrm{~h}$ & $8 \mathrm{~h}$ & $8 \mathrm{~h}$ & $8 \mathrm{~h}$ \\
Yield & $65 \%$ & $63 \%$ & $55 \%$ & $50 \%$ & $54 \%$ & $45 \%$ \\
\hline
\end{tabular}

3-(methylsulfonyl)-5-(2-((5-(1-(4-(trifluoromethyl)phenyl)-1H-imidazol5-yl)quinolin-8-yl)oxy)ethylidene)thiazolidine-2,4-dione(8a):

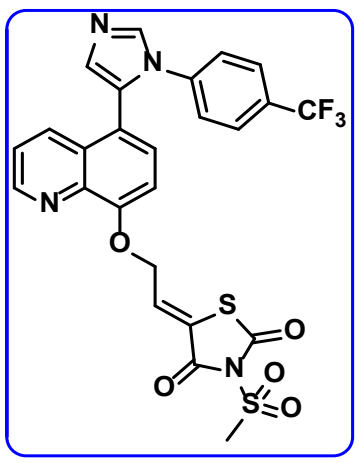

M.p: $280^{\circ} \mathrm{C}-283^{\circ} \mathrm{C}$. IR (KBr, cm-1): $3040,3025,1730,1645,1600,1320$, 1070, 770, 715 620. ${ }^{1} \mathrm{HNMR}$ ( $d_{6}$-DMSO, $\left.400 \mathrm{MHz}\right): 2.8$ (s, 3H), 4.67 (dd, $\left.1 \mathrm{H}\right)$, $4.68(\mathrm{dd}, 1 \mathrm{H}), 6.8(\mathrm{dd}, 1 \mathrm{H}), 7.1(\mathrm{~m}, 2 \mathrm{H}), 7.3(\mathrm{~d}, 2 \mathrm{H}), 7.6(\mathrm{~m}, 4 \mathrm{H}), 7.8(\mathrm{~d}, 1 \mathrm{H}), 8.4$ (d, $1 \mathrm{H}), 8.81(\mathrm{~d}, 1 \mathrm{H}) .{ }^{13} \mathrm{CNMR}$ ( $d_{6}$-DMSO, $400 \mathrm{MHz}$ ): 42, 64, 106, 121, 122, $123.5,124,124.5,126,130.5,132,135,138,139,145,148,164$ and 173.

3-(ethylsulfonyl)-5-(2-((5-(1-(4-(trifluoromethyl)phenyl)-1H-imidazol-5 -yl)quinolin-8-yl)oxy)ethylidene)thiazolidine-2,4-dione(8b):

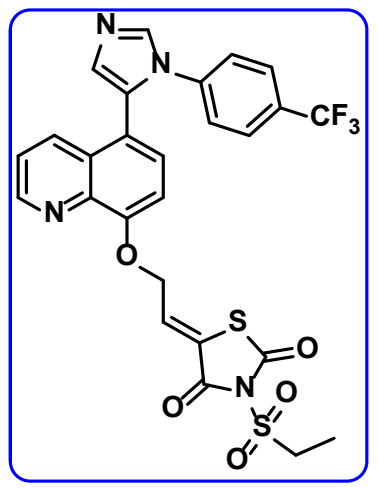

M.p: $288^{\circ} \mathrm{C}-290^{\circ} \mathrm{C}$. IR $\left(\mathrm{KBr}, \mathrm{cm}^{-1}\right): 3040,3025,1730,1645,1603,1325,1075$, 772, 715, 620. ${ }^{1} \mathrm{HNMR}\left(d_{6}\right.$-DMSO, $400 \mathrm{MHz}$ ): 1.3 (t, 3H), 3.45 (q, 2H), 4.65 $(\mathrm{dd}, 1 \mathrm{H}), 4.67(\mathrm{dd}, 1 \mathrm{H}), 6.8(\mathrm{dd}, 1 \mathrm{H}), 7.13(\mathrm{~m}, 2 \mathrm{H}), 7.32(\mathrm{~d}, 2 \mathrm{H}), 7.65(\mathrm{~m}, 4 \mathrm{H})$, $7.8(\mathrm{~d}, 1 \mathrm{H}), 8.41(\mathrm{~d}, 1 \mathrm{H}), 8.82(\mathrm{~d}, 1 \mathrm{H}) .{ }^{13} \mathrm{CNMR}\left(\mathrm{d}_{6}\right.$-DMSO, $\left.400 \mathrm{MHz}\right): 10,52$, $63,106,121,122,124,124.5,126,130,135,138,139,145,148,164$ and 173. 
3-tosyl-5-(2-((5-(1-(4-(trifluoromethyl)phenyl)-1H-imidazol-5-yl)quinoli n-8-yl)oxy) ethylidene) thiazolidine-2,4-dione(8c):

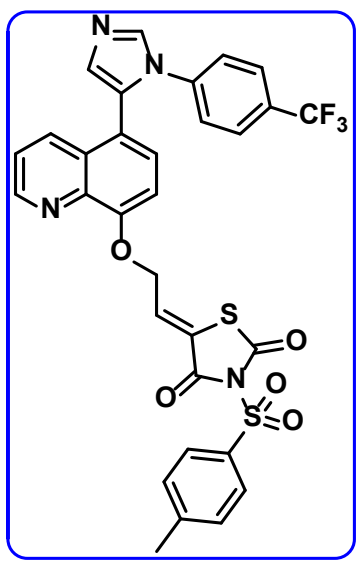

M.p. $300^{\circ} \mathrm{C}-302^{\circ} \mathrm{C}$. IR $\left(\mathrm{KBr}, \mathrm{cm}^{-1}\right): 3050,3030,1735,1640,1603,1325,1075$, 770, 715, 620. ${ }^{1} \mathrm{HNMR}\left(d_{6}\right.$-DMSO, $400 \mathrm{MHz}$ ): 2.3 (s, 3H), 4.68 (dd, 1H), 4.69 (dd, 1H), $6.8(\mathrm{dd}, 1 \mathrm{H}), 7.13(\mathrm{~m}, 2 \mathrm{H}), 7.32(\mathrm{~d}, 2 \mathrm{H}), 7.4(\mathrm{~d}, 2 \mathrm{H}), 7.65(\mathrm{~m}, 4 \mathrm{H}), 7.7$ $(\mathrm{d}, 2 \mathrm{H}), 7.8(\mathrm{~d}, 1 \mathrm{H}), 8.42(\mathrm{~d}, 1 \mathrm{H}), 8.85(\mathrm{~d}, 1 \mathrm{H}) .{ }^{13} \mathrm{CNMR}\left(d_{6}\right.$-DMSO, $\left.400 \mathrm{MHz}\right)$ : $20,64,107,121,122,123,124,124.5,126,128,130,132,133,135,138,139,145$, $148,155,165$ and 175 .

3-((4-chlorophenyl)sulfonyl)-5-(2-((5-(1-(4-(trifluoromethyl)phenyl)-1H -imidazol-5-yl)quinolin-8-yl)oxy)ethylidene)thiazolidine-2,4-dione (8d):

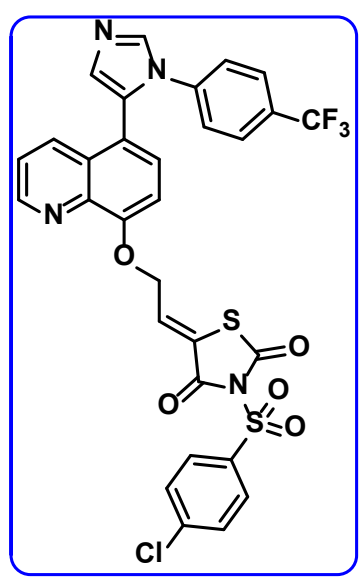

M.p: $290^{\circ} \mathrm{C}-292^{\circ} \mathrm{C} . \mathrm{IR}\left(\mathrm{KBr}, \mathrm{cm}^{-1}\right): 3070,3020,1720,1620,1530,1325,1080$, 770, 720, 655. ${ }^{1} \mathrm{HNMR}$ (d d -DMSO, $400 \mathrm{MHz}$ ): 4.68 (dd, H), 4.69 (dd, 1H), 6.85 (dd, 1H), $7.15(\mathrm{~m}, 2 \mathrm{H}), 7.28$ (d, 2H), $7.4(\mathrm{~d}, 2 \mathrm{H}), 7.63(\mathrm{~m}, 6 \mathrm{H}), 7.8(\mathrm{~d}, 2 \mathrm{H}), 7.95$ (d, $1 \mathrm{H}), 8.5(\mathrm{~d}, 1 \mathrm{H}), 8.87(\mathrm{~d}, 1 \mathrm{H}) .{ }^{13} \mathrm{CNMR}$ (d6-DMSO, $\left.400 \mathrm{MHz}\right): 64,108,121$, $122,123,124,124.5,126,128,130,132,133,134.7,135,137,139,145,149,155$, 162 and 173.

3-((4-bromophenyl)sulfonyl)-5-(2-((5-(1-(4-(trifluoromethyl)phenyl)-1H -imidazol-5-yl)quinolin-8-yl)oxy)ethylidene)thiazolidine-2,4-dione (8e): 


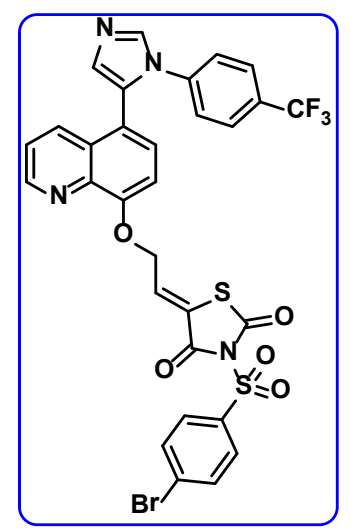

M.p: $298^{\circ} \mathrm{C}-300^{\circ} \mathrm{C}$. IR $\left(\mathrm{KBr}, \mathrm{cm}^{-1}\right): 3080,3025,1720,1623,1530,1325,1085$, 770, 725, 655. ${ }^{1} \mathrm{HNMR}\left(d_{6}\right.$-DMSO, $\left.400 \mathrm{MHz}\right): 4.68(\mathrm{dd}, 1 \mathrm{H}), 4.69(\mathrm{dd}, 1 \mathrm{H}), 6.87$ (dd, $1 \mathrm{H}), 7.18(\mathrm{~m}, 2 \mathrm{H}), 7.28(\mathrm{~d}, 2 \mathrm{H}), 7.6(\mathrm{~m}, 4 \mathrm{H}), 7.88(\mathrm{~m}, 5 \mathrm{H}), 8.4(\mathrm{~d}, 1 \mathrm{H}), 8.8$ (d, $1 \mathrm{H}$ ). ${ }^{13} \mathrm{CNMR}\left(d_{6}\right.$-DMSO, $\left.400 \mathrm{MHz}\right): 64.2,107,121.8,122.4,123.8,123.9$, $124,124.2,124.5,126,128,130,131.8,132,134.5,136,139,146,149,155,163$ and 174 .

3-((4-nitrophenyl)sulfonyl)-5-(2-((5-(1-(4-(trifluoromethyl)phenyl)-1H-i midazol-5-yl)quinolin-8-yl)oxy)ethylidene)thiazolidine-2,4-dione(8f):

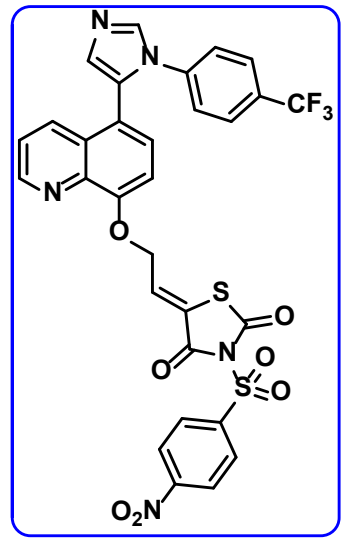

M.p: $305^{\circ} \mathrm{C}-308^{\circ} \mathrm{C} . \mathrm{IR}\left(\mathrm{KBr}, \mathrm{cm}^{-1}\right): 3070,3028,1720,1623,1535,1400,1328$, 1085, 770, 730, 655. ${ }^{1} \mathrm{HNMR}\left(d_{6}\right.$-DMSO, $400 \mathrm{MHz}$ ): 4.68 (dd, 1H), 4.69 (dd, $1 \mathrm{H}), 6.87(\mathrm{dd}, 1 \mathrm{H}), 7.18(\mathrm{~m}, 2 \mathrm{H}), 7.28(\mathrm{~d}, 2 \mathrm{H}), 7.6(\mathrm{~m}, 4 \mathrm{H}), 7.8(\mathrm{~d}, 1 \mathrm{H}), 8.12(\mathrm{~d}$, $2 \mathrm{H}), 8.4(\mathrm{~m}, 3 \mathrm{H}), 8.85(\mathrm{~d}, 1 \mathrm{H}) .{ }^{13} \mathrm{CNMR}\left(d_{6}\right.$-DMSO, $\left.400 \mathrm{MHz}\right): 64.2,107,121$, $122,123.8,124,124.5,124.8,126,128,130,132,134,136,139,142,146,149,151$, 155.4, 164 and 174.5.

\section{Conclusion}

In current research work, we successfully synthesized and characterized Mannich bases of quinoline attached imidazoline containing thiozolidine-2,4-dione derivatives and sulfonamide analogues. In step 2, cyclization was achieved in good yield. In step 4 , aldehyde was isolated with good yield. Step $7 \& 8$ avoid te- 
dious work-up and gain good yields. We strongly believe this is a novel route for synthesis of Mannich bases of thiozolidne-2,4-dione and sulfonamide analogues. We are planning to make these derivatives check for biological evolution and details will include in next journal.

\section{Acknowledgements}

I sincerely thank my guide, co-workers, and Department of Chemistry, Sri Krishnadevaraya University (Anantapur, Andhra Pradesh, India) and Gvk Bio sciences. Pvt. Ltd. for providing laboratory and analytical facilities.

\section{References}

[1] Vasincu, I.M., Apotrosoaei, M. and Panzariu, A.-T. (2014) Synthesis and Biological Evolution of New 1,3-Thiozolidine-4-one Derivatives of 2-(4-Isobutyl phenyl) Propionic Acid. Molecules, 19, 15005-15025.

[2] Chadha, N., Bahia, M.S., Kaur, M. and Silakari, O. (2015) Thiazolidine-2,4-Dione Derivatives: Programmed Chemical Weapons for Key Protein Targets of Various Pathological Conditions. Bioorganic \& Medicinal Chemistry, 23, 2953-2974. https://doi.org/10.1016/j.bmc.2015.03.071

[3] Sharghia, H., Khalifeha, R., Moeinia, B.F., Beyzavia, M.H., Salimi Benic, A. and Doroodmand, M.M. (2011) Mannich Reactions of Secondary Amines, Aldehydes and Alkynes in Water using $\mathrm{Cu} / \mathrm{C}$ Nanoparticles as a Heterogenous Catalyst. Journal of the Iranian Chemical Society, 8, S89-S103.

[4] Kapoor, A. and Khare, N. (2016) Antibacterial and Antifungal Evaluation of Mannich Bases of 2,4 Thiazolidinedione and Rhodanine. Der Pharmacia Lettre, 8 , 143-148.

[5] Panzariu, A.-T., Aprotosoaei, M. and Profire, L. (2016) Synthesis and Biological Evaluation of new 1,3-Thiazolidine-4-one Derivatives of Nitro-1-Arginine Methyl Ester. Chemistry Central Journal, 10, 6.

[6] Shaikh, F.M., Patel, N.B. and Rajani, D. (2013) Synthesis of New Thiazolidine-2,4,Dione Derivatives \& Their Antimicrobial \& Antitubercular Activity. Indian Journal of Research in Pharmacy and Biotechnology, No. 1, 496-503.

[7] Prakash, D. and Bhoi, U.A. (2011) A Complete Review of Thiazolidine-4-One. Journal of Pharmacy Research, 4, 2436-2440.

[8] Previtera, T., Mervigorita, V. and Fenech, G. (1990) 3,3'-Di(1,3-Thiazolidine4-One) System. Synthesis and Pharmacological Properties of 3,3'(1,2-Ethanediyl) Bis-(2-Hetero aryl-1,3-Thiazolidine-4-One) Derivatives. European Journal of Medicinal Chemistry, 25, 569-579.

[9] Patel, K.D., Patel, C.N. and Patel, G.M. (2016) Synthesis and Evaluation of Some New Thiazolidin-4-One Derivatives as Potential Antimicrobial Agents. Med-Chem (Los Angeles), 6, 10.

[10] Singh, T. and Khobragade, D. (2014) Synthesis and Evaluation of Thiazolidine-4-One for Their Antibacterial Activity. JPSBR, 4, 110-113.

[11] Bhaviskar, B.A., Khadabadi, S.S. and Deore, S.L. (2013) Microwave Assisted Synthesis and Diabetic Activity of Novel 5-[4-(Substituted) Benzylidine] Thiozolidine-2,4-Dione. Journal of Chemistry, 2013, Article ID: 656271.

[12] Primas, N., Verhaeghe, P., Cohen, A., Kieffer, C., et al. (2012) A New Synthetic Route to Original Sulfonamide Derivatives in 2-Trichloromethylquinazoline Series: 
A Structure-Activity Relationship Study of Antiplasmodial Activity. Molecules, 17, 8105-8117. https://doi.org/10.3390/molecules17078105

\section{Abbreviations and Acronyms}

DMF: Dimethylformamide

EtOAc: Ethyl Acetate

DCM: Dichloromethane

DIPEA: N,N-Diisopropylethylamine

$\mathrm{NaH}$ : Sodium hydride

TosMIC: Toluenesulfonylmethyl Isocyanide

Submit or recommend next manuscript to SCIRP and we will provide best service for you:

Accepting pre-submission inquiries through Email, Facebook, LinkedIn, Twitter, etc. A wide selection of journals (inclusive of 9 subjects, more than 200 journals)

Providing 24-hour high-quality service

User-friendly online submission system

Fair and swift peer-review system

Efficient typesetting and proofreading procedure

Display of the result of downloads and visits, as well as the number of cited articles Maximum dissemination of your research work

Submit your manuscript at: http://papersubmission.scirp.org/

Or contact ijoc@scirp.org 